\title{
Automated region detection based on the contrast-to-noise ratio in near-infrared tomography
}

\author{
Xiaomei Song, Brian W. Pogue, Shudong Jiang, Marvin M. Doyley, Hamid Dehghani, \\ Tor D. Tosteson, and Keith D. Paulsen
}

\begin{abstract}
The contrast-to-noise ratio (CNR) was used to determine the detectability of objects within reconstructed images from diffuse near-infrared tomography. It was concluded that there was a maximal value of CNR near the location of an object within the image and that the size of the true region could be estimated from the CNR. Experimental and simulation studies led to the conclusion that objects can be automatically detected with CNR analysis and that our current system has a spatial resolution limit near $4 \mathrm{~mm}$ and a contrast resolution limit near 1.4. A new linear convolution method of CNR calculation was developed for automated region of interest (ROI) detection. (C) 2004 Optical Society of America

OCIS codes: $170.6960,170.7050,110.2960,110.3000,110.6960,170.3830$.
\end{abstract}

\section{Introduction}

Because near-infrared (NIR) diffuse tomography can noninvasively quantify oxygen saturation, hemoglobin concentration, water concentration, scattering, and potentially exogenous chromophores, it may provide an effective diagnostic tool for early detection of breast cancer. ${ }^{1-6}$ Tumors have a higher level of vascularity due to hyperactive angiogenesis leading to optical contrast to regular tissues in the NIR spectrum or light between 650 and $900 \mathrm{~mm} .{ }^{7,8}$ Detecting these changes with optical absorption and scatteringbased tomography requires implementation of accurate and fast image reconstruction algorithms. Because the reconstruction process results in moderate-resolution images, it is becoming increasingly important to develop tools to accurately interpret the reconstructed image and promptly find the shape, size, and location of the tumor or the region of interest (ROI).9-11 Even more objectively, it would be desirable to automatically find regions of increased

X. Song, B. W. Pogue (pogue@dartmouth.edu), S. Jiang, M. M. Doyley, H. Dehghani, and K. D. Paulsen are with the Thayer School of Engineering, Dartmouth College, Hanover, New Hampshire 03755. M. M. Doyley is also with the Department of Diagnostic Radiology, Dartmouth Medical School, Hanover, New Hampshire 03755. T. D. Tosteson is with Biostatistics, Norris Cotton Cancer Center, Dartmouth-Hitchcock Medical Center, Lebanon, New Hampshire 03756.

Received 28 June 2003; revised manuscript received 27 October 2003; accepted 28 October 2003.

0003-6935/04/051053-10\$15.00/0

(C) 2004 Optical Society of America contrast and quantify their values. However, in most of current preclinical and clinical NIR tomography studies, the shape and location of the ROI in the reconstructed image are decided by a radiologist projecting what is observed in the x-ray mammograms into the circular tomographic coordinate system of the NIR tomography images. Although this approach is practical and works reasonably well, it has some subjectivity associated with it as well as problems associated with tissue deformation. This approach becomes especially problematic when one considers that the image reconstruction process for NIR tomography is nonlinear and has its own unique set of artifacts and a spatially dependent contrastdetail response. ${ }^{12,13}$ As NIR tomography transitions into a tool for imaging patients, a quantitative computational tool is necessary for consistent and fast ROI detection and delineation to eliminate use of subjective ROI detection. Furthermore, because evaluating a new imaging modality requires knowledge of the validity of the reconstructed images, this type of computational tool can be used to generate contrast-detail curves that can be used to objectively determine the system performance and allow accurate comparison with other imaging systems.

To develop an accurate and efficient computational tool for automatically and efficiently segmenting out the ROI in a laboratory or clinical NIR tomography imaging system, an automated algorithm to calculate the contrast-to-noise ratio (CNR) is used. Similar to the signal-to-noise ratio in digital signal-processing theory, the CNR is defined as the difference between the ROI and the background region values of the 
optical properties (i.e., the absorption and reduced scattering coefficients) divided by the average variation in the background, providing a relative difference property value. Although there is only one choice for the ROI location, there are different choices for the background that can be used for the analysis. Ideally, the property distribution of the reconstructed image should be exactly the same as the real image whose background (or regular tissue) is uniform and identical everywhere. It is reasonable to randomly choose a ROI within the whole image. However, the intrinsic diffusion characteristic of the light traveling in the tissue makes every spot of the reconstructed image dependent on other locations of the same image, and generally there is a radial variation in the modulation transfer response function. ${ }^{12}$ Thus it is equally justified for one to consider taking background values from the same radial locations of the ROI to avoid biasing the analysis to regions with a different spatial-frequency response. We need to take this into account and find the best solution for calculating the background properties. In this study we investigated and compared three methods of choosing the background values, and we provide our results in this paper.

A contrast-detail curve, where the line of minimum-detected contrast is plotted for varioussized inclusions located within a tissue, is an accurate and objective way to evaluate the spatial resolution of a medical image system, especially in computed tomography and ultrasound. ${ }^{13-17}$ It indicates the transition from a detectable heterogeneity to a statistically insignificant undetectable heterogeneity, and the shape of the curve provides a quantitative description of the system's performance and is particularly relevant where the clinical role of the system is in the regime of a low-contrast or lowresolution modality. Traditionally, contrast-detail curves are derived subjectively by human inspection, ${ }^{18}$ but it is reasonable to expect some interobserver variability in the development of these curves. Thus, as a first attempt in this direction, we used an objective threshold as the measure of detection rather than using repeated observers. Given a chosen threshold value of CNR, then it is fairly straightforward to accurately calculate the CNR values of reconstructed images and find those images in which objects appear to be detectable. Thus, based on whether the object presence is above or below the threshold, a contrast-detail curve can be created that shows the minimum contrast required to detect objects at each size (i.e., detail is used for size in this types of resolution test), with the assumption that a CNR of 4 is required for detection of the object. The justification for this threshold level and the implications for clinical utility are discussed.

\section{Materials and Methods}

\section{A. Image Reconstruction}

NIR frequency-domain absorption and scatter tomography is based on a finite-element method reconstruc-

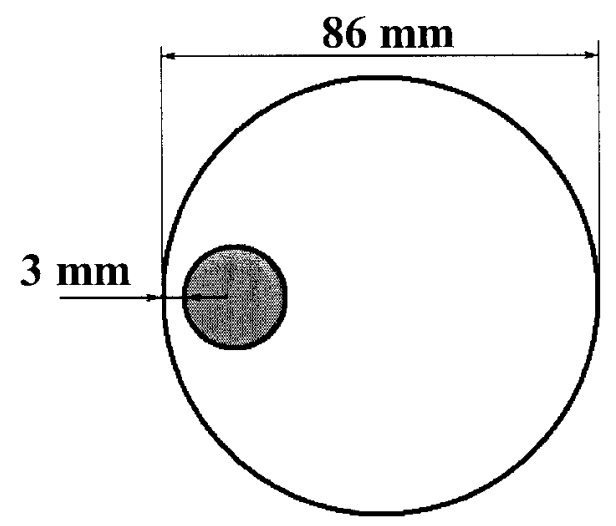

(a)

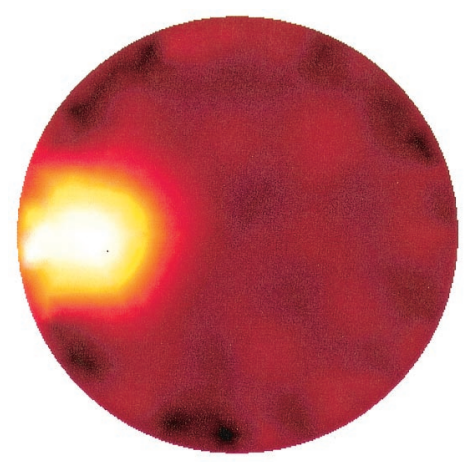

(b)

Fig. 1. (a) Geometry of the simulation for optical property distribution, and (b) a typical reconstructed absorption coefficient image. The image in (b) was generated with simulated forward data with a $1 \%$ noise in amplitude and a $1^{\circ}$ noise phase shift by use of zero-mean Gaussian distribution.

tion algorithm and produces three-dimensional images of absorption and scatter from boundary measurements of light transport through tissue. ${ }^{19}$ For this paper, we choose a circular geometry of $86 \mathrm{~mm}$ in diameter for our simulation studies. The background has an absorption coefficient $\mu^{a}=0.005$ $\mathrm{mm}^{-1}$ and a reduced scattering coefficient $\mu_{s}{ }^{\prime}=1.0$ $\mathrm{mm}^{-1}$. Within this field, a spherical heterogeneity with a variable diameter was located $3 \mathrm{~mm}$ away from the edge as depicted in Fig. 1, with a fixed $\mu_{s}{ }^{\prime}=$ $1 \mathrm{~mm}^{-1}$ and a variable $\mu_{a}$ to provide the required contrast in absorption from 1.2:1 to 5.0:1. We accomplished the forward calculations of diffusion theory with a finite-element numerical solution using a circular mesh with a set of regularly spaced node positions. Noise can be added to either the simulated measured data or the optical property distribution. In this study, zero-mean Gaussian noise of $1 \%$ in amplitude and $1 \mathrm{deg}$ in phase shift were added to the calculated boundary data to simulate the realistic condition area in our NIR system. ${ }^{20}$ It is well known that diffuse imaging reconstruction methods are in general ill-posed; the simulation can be used to test the accuracy of the inverse solution or to provide 
interactive criteria and information that are useful to improve the reconstruction algorithm. With the image reconstruction process, we use a NewtonRaphson approach to iteratively solve for the spatial distribution of optical properties, which minimizes the squared error between sets of measured (simulated) and calculated data. In the reconstruction process, the goal is recovery of $\mu_{a}$ and $\mu_{s}{ }^{\prime}$ at each finite-element method node based on simulated or real measurements of light irradiance at the tissue surface. Without an analytic inverse solution, the computational way of finding this inverse solution is to minimize the difference between data from the tissue surface and the calculated data from the forward solver. A Levenberg-Marquardt-style regularization algorithm was used to step down the regularization parameter as the number of iterations progressed. ${ }^{20,21}$

\section{B. Phantom Studies}

To test the spatial resolution of our NIR imaging system, a solid phantom was used to mimic the optical properties that are described in Section 4. The details of this type of phantom and its use has been described in detail in a previous paper. ${ }^{21}$ The imaging system at Dartmouth College uses amplitudemodulated light at $100 \mathrm{MHz}$ generated from six commercial laser diodes that span the NIR spectral range as shown in Fig. 2. The amplitude-modulated light is focused into a fiber that is coupled to each laser diode mount (not shown in Fig. 2 for clarity). A radio-frequency switch is used to sequence the sources on and off one at a time under computer control while a fiber-optic combiner delivers the selected light to a single-source fiber interfaced to the phantom under test. The generated light is directed into the phantom (or breast) through a fiber-optic array guided by a precision positioning system. Sixteen photomultiplier tubes (PMTs) circularly mounted on an aluminum disk are multiplexed in parallel to each of the 16 detection fibers. This configuration allows injection of a source of light into one of the 16 fibers, while the other 15 bundles remain connected to PMTs for light detection. This unique arrangement was designed to allow the PMTs to measure from all 16 source positions without changing detector gain, thereby minimizing the time required for data acquisition. ${ }^{16}$ The cylindrical solid phantom is approximately $84 \mathrm{~mm}$ in diameter and $90 \mathrm{~mm}$ in height, with an absorption coefficient $\mu_{a}=0.0044$ $\mathrm{mm}^{-1}$ and a reduced scattering coefficient $\mu_{s}{ }^{\prime}=1.03$ $\mathrm{mm}^{-1}$ in the background. A circular hole of $4 \mathrm{~mm}$ in diameter, which is the spatial resolution as discussed in Subsection 4.c, was cut at $3 \mathrm{~mm}$ away from the phantom edge, as shown in Fig. 1. A solution with Intralipid and India ink was filled into the target hole to produce relevant absorption coefficient contrasts ranging from $2: 1$ to $9: 1$ with a closely matched scattering coefficient to the solid phantom. The phantom was imaged at a 785-nm wavelength by our NIR imaging system, and the experimental data were then reconstructed by the same diffuse imaging re-

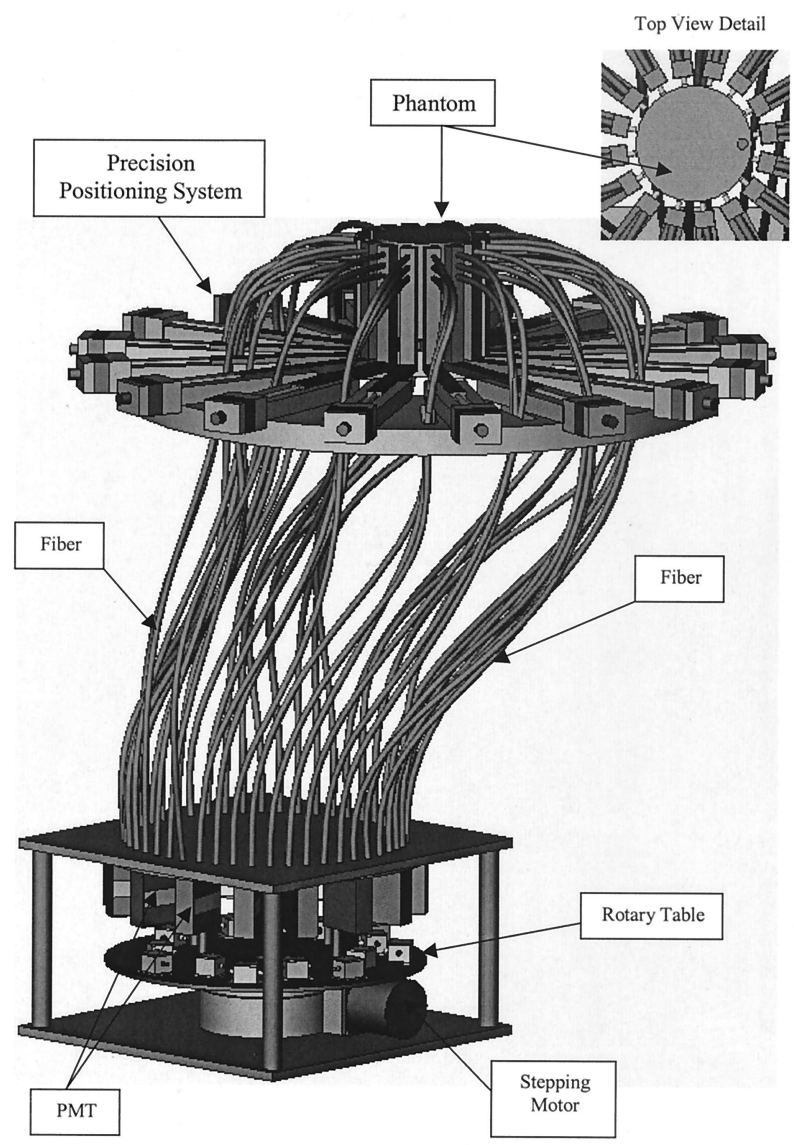

Fig. 2. NIR tomographic imaging system (partial view).

construction method used in the simulation. We used the two-dimensional (2-D) linear convolution method to calculate the maximum CNR and to find the locations of the ROIs on the absorptionreconstructed images of different absorption contrasts.

\section{Theory}

\section{A. Definitions of Contrast-to-Noise Ratio}

CNR is defined as the difference between the averaged optical coefficient within the ROI and the differences within the background region, divided by the averaged optical coefficient variation in the background. Because the background profile of the ROI is unknown after forward calculation as well as in real experimentally acquired data, one of the goals of this study is to find the proper way to calculate the background profile. We developed three methods of choosing the relevant background values to calculate the CNR and construct the contrast-detail curves in this study. The three choices were (i) backgrounds sampled at the same radial distance in the image, (ii) backgrounds of randomly sampled ROIs of the same size as the original, and (iii) all the available background. First, the background profile we chose in the image field had the same radial size and similar location of the target, and they did not overlap each other as shown in Fig. 3(a); thus they had indepen- 


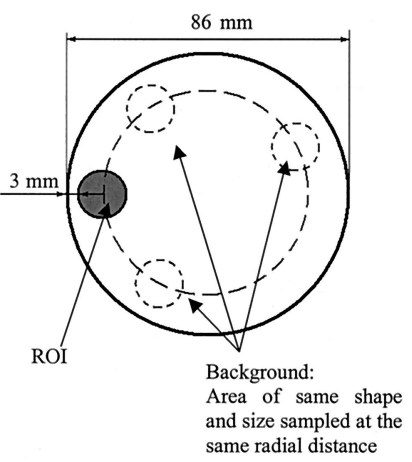

(a)

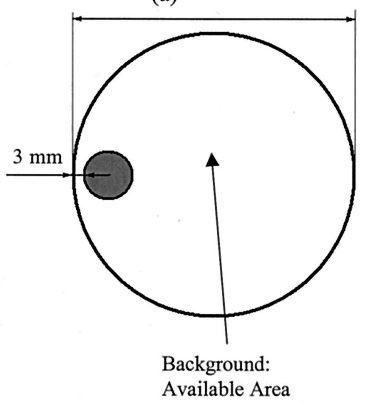

(c)

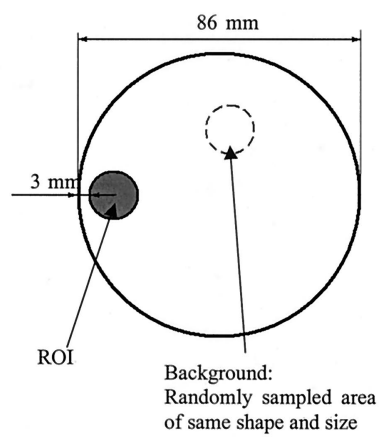

(b)
Fig. 3. Schematic showing the three possible choices of backgrounds: (a) sampled at the same radial location as the lesion object, (b) randomly sampled, or (c) sampled by use of the entire region outside of the target lesion object.

dent mean values and noises. As many nonoverlapping ROI-shaped areas as possible were chosen to minimize the displacement error.

We can define the contrast in the reconstructed image as the difference of the mean values in the target and background; CNR can be defined as

$$
\mathrm{CNR}_{\mathrm{I}}=\frac{\text { contrast }}{\text { noise }}=\frac{\Delta \mu}{\text { noise }}=\frac{\mu_{\mathrm{ROI}}-\mu_{\text {mean }}}{\left[\left({\sigma_{\mathrm{ROI}}}^{2}+\sigma_{\text {mean }}^{2}\right) / 2\right]^{1 / 2}},
$$

where $\mu_{\mathrm{ROI}}$ is the mean of the node values in the target; $\mu_{\text {mean }}$ is the mean value of the averaged node values in the total ROI-shaped background areas; and $\sigma_{\mathrm{ROI}}$ and $\sigma_{\text {mean }}$ are the standard deviations of the collectivities of the target and the mean of the background areas, respectively. Because the circular simulated target and background areas have the same size, it is reasonable to assume that the target and background areas have the same noise weights.

However, the noise from the nodes that are closest to the field edge is higher because of the nonlinear image reconstruction process used here and because the weight functions (i.e., Jacobian matrices) have much higher contributions nearest the source and detector locations. Overall, this contributes to higher system noise in regions nearest the edge of the field and lower values toward the center of the field. This effect is not accounted for in the above definition of CNR. As shown in Fig. 3(b), to make sure that every node in the background has an equal chance to contribute to the background noise value, we introduce the second definition of CNR as

$$
\mathrm{CNR}_{\mathrm{II}}=\frac{\mu_{\mathrm{ROI}}-\mu_{\text {random }}}{\left[\left({\sigma_{\text {ROI }}}^{2}+\sigma_{\text {random }}^{2}\right) / 2\right]^{1 / 2}},
$$

where $\mu_{\text {random }}$ is the mean value of the nodes within a region having a location that is randomly chosen in the variable background and $\sigma_{\text {random }}$ is the standard deviation of these same nodes. Only one location of the ROI-shaped area was chosen in one calculation, such that the ROI background profile is truly random. Although this definition has a good basis in statistical probability theory, the shortcoming of this definition is that the CNR depends on randomly chosen nodes in the whole image; therefore CNR is not exactly identical among different calculations.

In the first two definitions of CNR, ROI and the testing background have same area; thus a noise weight, which presents the contribution of a certain area noise to the whole area noise, is not necessary. But if we use the whole available area as the testing background, as shown in Fig. 3(c), we must include a noise weight into the CNR definition because the background and ROI have different contributions to the whole noise. The CNR than becomes

$$
\mathrm{CNR}_{\mathrm{III}}=\frac{\mu_{\mathrm{ROI}}-\mu_{\text {background }}}{\left[w_{\mathrm{ROI}} \sigma_{\mathrm{ROI}}{ }^{2}+w_{\text {background }} \sigma_{\text {background }}{ }^{2}\right]^{1 / 2}},
$$

where $\mu_{\text {background }}$ is the mean value over the variable background and $w_{\mathrm{ROI}}=\mathrm{AREA}_{\mathrm{ROI}} /\left(\mathrm{AREA}_{\mathrm{ROI}}{ }^{+}\right.$ $\left.\mathrm{AREA}_{\text {background }}\right)$, and $w_{\text {background }}=\mathrm{AREA}_{\text {background }} /$ $\left(\mathrm{AREA}_{\mathrm{ROI}}{ }^{+} \mathrm{AREA}_{\text {background }}\right)$ are the noise weights. We use this simple linear form of noise weight because the exact noise weight of different areas in the field is hard to determine because of the nonlinearity of the inverse problem.

\section{B. Detection of the Location of the Region of Interest} in the Reconstructed Image

Estimates of the locations of the ROI in the reconstructed images are necessary in our simulation and experimental studies. Two methods were used to detect the location of the ROI with different sizes and absorption contrast values where the step size for scanning was 0 to $5 \mathrm{~mm}$. In the first method, it was assumed that the CNR should be the maximum when the suppositional region was at the location of the ROI, as shown in Fig. 4(a). In this method, CNR was calculated by the third method defined in Eq. (3), which includes all the available background as the background area. However, this algorithm requires a significant period of time to complete the calculations for all possible locations and sizes of the ROI within the field. Thus a 2-D linear convolution method was introduced to detect the ROI. In this method, a ROI pattern that has the same optical coefficient profile as the simulated lesion in computer simulations or a phantom study was created, and we developed the correlation image by convolving it with the reconstructed image in which the highest corre- 


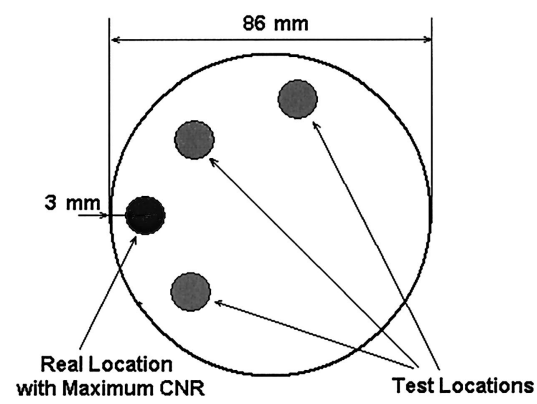

(a)

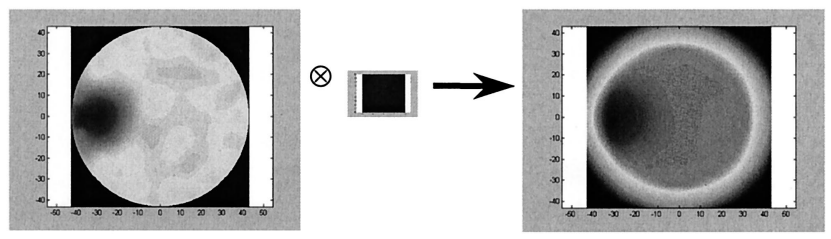

(b)

Fig. 4. Two methods of ROI detection: (a) Detection by a search for the maximum CNR from all the possible locations in the image field where alternate possible locations are randomly tested and the sizes of these regions are varied. This approach is used to determine when the CNR calculation is maximal and yields an estimate of the size and location of the ROI but requires a significant amount of computational time to achieve the result. (b) 2-D linear convolution method where $\otimes$ denotes the convolution process.

lation location corresponds to the maximum CNR, as depicted in Fig. 4(b). Comparing this approach with the previous method, the advantage of the 2-D linear convolution method is that it decreases the calculation time from several minutes to a few seconds for processing one image.

\section{Results}

\section{A. Evaluating the Three Methods of Calculating the Contrast-to-Noise Ratio}

Assuming that the real diameter and location of the target are given, we calculated the CNR by using the three methods described above as we systematically varied the absorption contrast and the ROI size. Figure 5 shows the difference in the CNR values of the three methods in the simulation study. Figure $5(a)$, in which we used the first method of calculating the ROI background profile, is the CNR distribution for different ROI sizes and absorption contrasts; for each ROI diameter value, the CNR was calculated and assigned to the related spot in the CNR profile as the absorption contrast varies from 0 to 5 . The resolution is $1 \mathrm{~mm}$ for the horizontal axis and 0.2 for the vertical axis. Similarly, the CNR distribution profiles of the second and third background calculating methods are plotted in Figs. 5(b) and 5(c). Theoretically, if we apply a practical detection threshold with a CNR of 4 , which indicates that any reconstructed image with a CNR lessthan 4 is not detectable to the human eye, we can find the transition from a detectable ROI to a nondetectable ROI on any of the profiles plotted in Figs. 5(a)-5(c). In practice, the transition boundary points around where the CNR transits from smaller than 4 to larger than 4 were calculated and served as the construction points to plot the contrast-detail curve. The interpolation method was used to find and smooth the transition boundary. As we can see from Figs. 5(a)-5(c), the CNR profile calculated by the third method is apparently the best profile to use to find the transition boundary, as shown in Fig. 5(d). The contrast-detail curve in Fig. 5(d) shows the spatial resolution of the NIR breast imaging system used in our study.

Our choice of a CNR of 4 as the detection threshold was based on the simulation study performed on different ROI size and contrast combinations. As shown in Fig. 6, both the target with a large size (23 $\mathrm{mm}$ in diameter) and low contrast (1.4 in absorption) and the target with the small size (3 $\mathrm{mm}$ in diameter) and high contrast (3.6 in absorption) can be observed in the reconstructed images if the CNR is greater than 4 for all three calculation methods. Thus it is reasonable to set the CNR to 4 as the detection threshold, although clearly a more-indepth study should be undertaken to determine which CNR value would best correspond to that used by humans. Nonetheless for this study we used a CNR of 4 as a possible threshold. The actual CNR values are plotted for all sizes and absorption contrast, as shown in Figs. 5(a)-5(c) as well as the system contrastdetail curve Fig. 5(d) assuming a decision threshold CNR of 4 .

\section{B. Detection of the Location of the Region of Interest}

As discussed above we used both the node-by-node method, in which the maximum CNR from all the suppositional regions in the image field is searched, and the 2-D linear convolution method, in which the maximum correlation location between the ROI pattern and the reconstructed image profile was detected. In both methods we calculated the CNR by Eq. (3), and the differences between the true location of the ROI and its detected location were compared at different contrast values, as shown in Fig. 7.

Figure 7 shows that the error between the detected and the true locations of the ROI is even larger than the size of the ROI in both detection methods if the diameter of the target is less than $3 \mathrm{~mm}$ and the contrast is low [i.e., equal to 2 in Fig. 7(a)]. As the contrast increases [Fig. 7(b)-7(d)], the location error from the node-by-node matching method is less than $1 \mathrm{~mm}$, but it requires a maximum CNR much lower than 4 - the CNR threshold we assumed above. The error from the 2-D linear convolution method is also large when the diameter of the ROI is small. Thus neither of the two detection methods can find the location of the ROI if the diameter of heterogeneity is less than $3 \mathrm{~mm}$. As the diameter of the ROI becomes greater than $4 \mathrm{~mm}$, the location errors from both of the methods are around 1-2.5 mm. Although the 


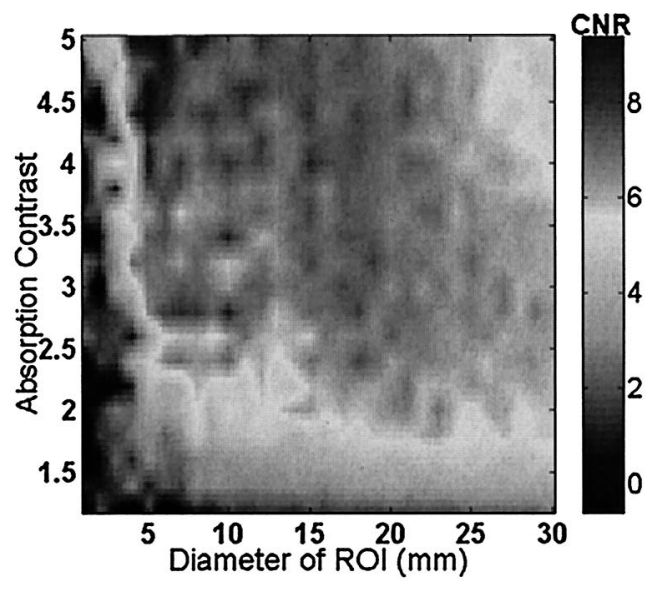

(a)

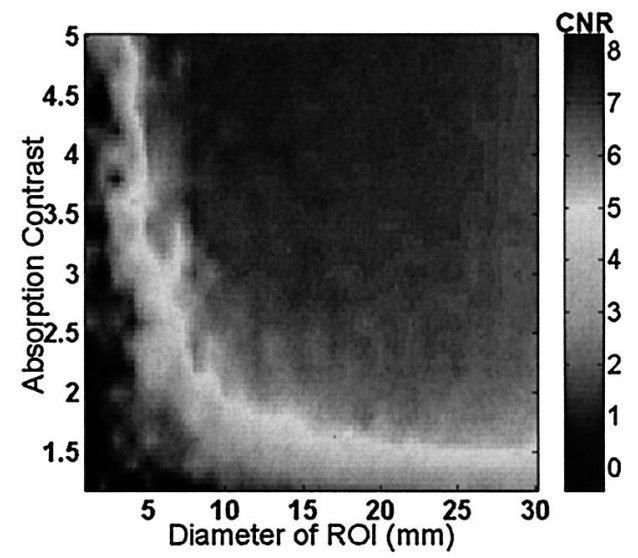

(c)

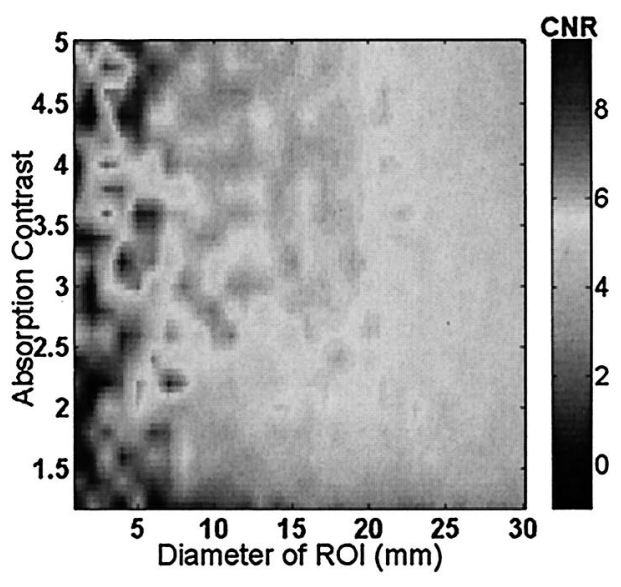

(b)

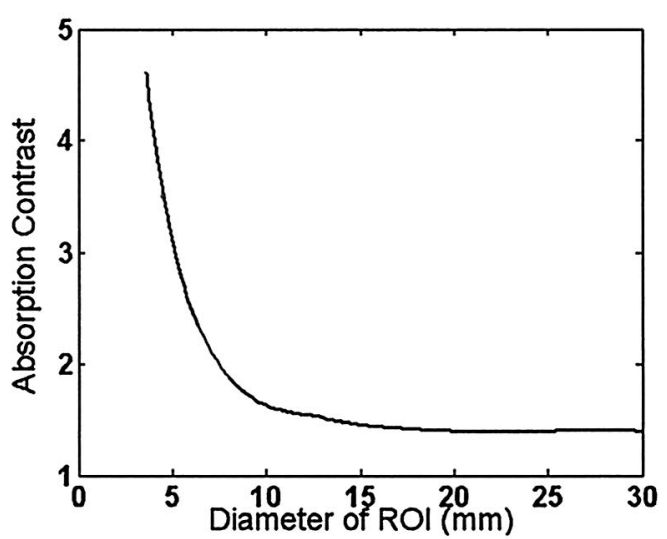

(d)

Fig. 5. Graphs of CNR distribution (the color bar represents CNR values) obtained by the three different methods of choosing the background areas including (a) $\mathrm{CNR}_{\mathrm{I}}$, (b) $\mathrm{CNR}_{\mathrm{II}}$, and (c) $\mathrm{CNR}_{\mathrm{II}}$. images are plotted in by a detection threshold with a CNR of 4 .

(d) Based on the method shown in (c), the contrast-detail curve for the

location error from the convolution method is slightly larger, it is more than ten times faster.

\section{Phantom Study Results of Spatial Resolution}

The photograph of the actual phantom we used in our study is shown in Fig. 8. The 4-mm-diameter hole was filled with an Intralipid and ink solution with a variable absorption contrast from $2: 1$ to $9: 1$ and a closely reduced scattering coefficient with the phantom. Figure 9 shows the reconstructed absorption images with the absorption contrast values of 2.0, 4.0, 6.0 , and 9.0, respectively, and their maximum CNR values are $2.9,3.8,4.9$, and 5.8 calculated by the third definition of CNR. The noise in the experiment includes not only the random noise but also the systematic noise. ${ }^{10}$ The random noise in the experimental measurement is typically from the stochastic detector fluctuations, which is almost equal to or a little less than $1 \%$ of the noise level that we added to the calculated boundary data in our simulation study. As an indication, a comparison of these results with the results in the simulation, we can see that there are more obvious artifacts in the reconstructed images of the phantom study, especially at the edge of the field. However, we still can observe that the reconstructed positions of the ROI shift around $2 \mathrm{~mm}$ toward the edges of the field, as discussed above. Also we can see that, if the CNR values are higher than 4, the 4-mm-diameter object is clearly visible on top of the noisy background (detectable by observers or by the computer) as shown in Figs. 9(c) and 9(d). But if the CNR value is lower than 3.0, as indicated in Fig. 9(a), even the computer cannot detect the ROI location by any of our location detection methods. Thus the detectable size of our NIR imaging system is at least $4 \mathrm{~mm}$, providing a good match to the results we achieved in our simulation. 


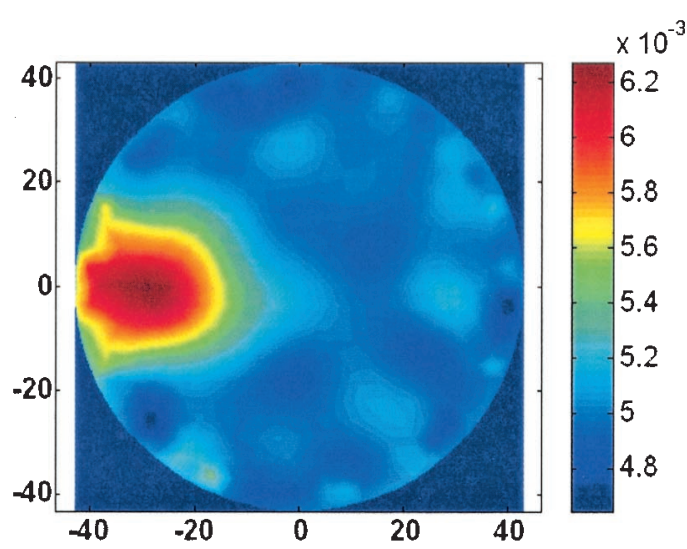

(a)

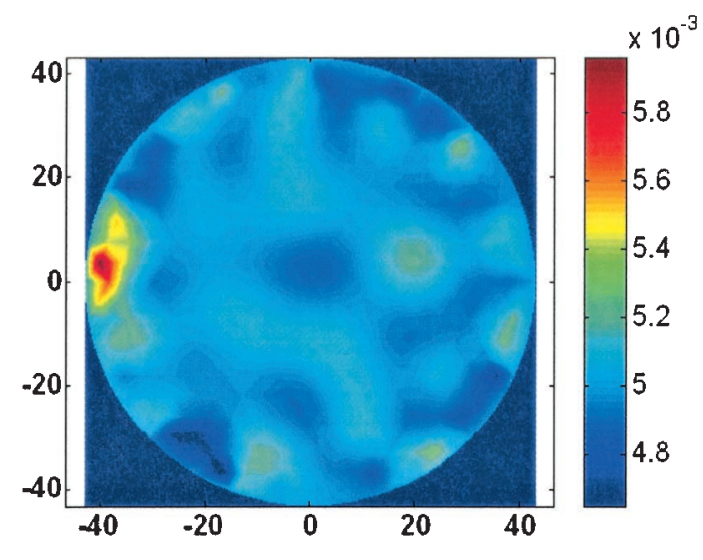

(b)

Fig. 6. Reconstructed absorption images for (a) the target with the large size (23 mm in diameter) and low contrast (1.4) and (b) the target with the small size ( $3 \mathrm{~mm}$ in diameter) and high contrast (3.6) in which CNR is greater than 4 by all three calculation methods.

\section{Discussion}

Comparing our three CNR calculation methods, of the graphs in Fig. 5 illustrate that the choice of background value can significantly effect the decision criteria of whether the region is detected. The first definition of CNR that was based on the matched filter method did not include information about the nodes closest to the edge of the image field. This contributed to a higher noise because of the effect of spatially dependent noise in NIR tomographic images, where most high-frequency noise is located in the periphery of the image and lower-frequency noise is located in the interior of the image. In the second

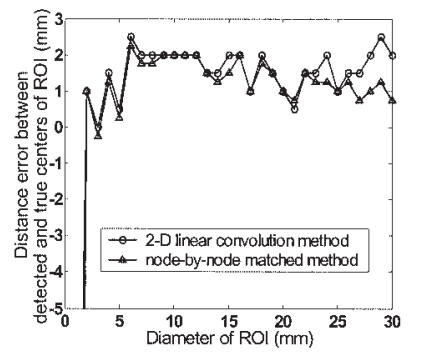

(a)

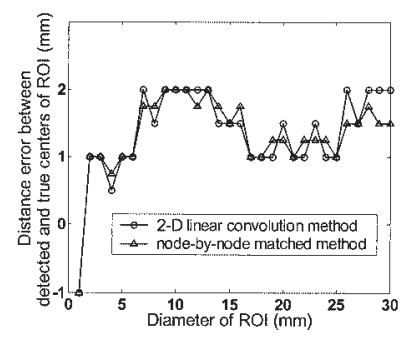

(b)

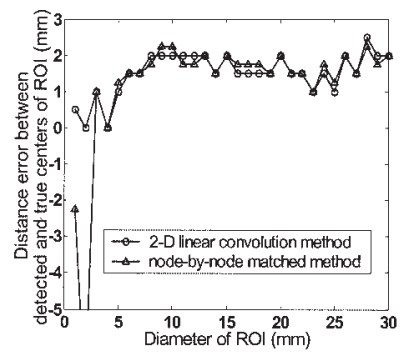

(b)

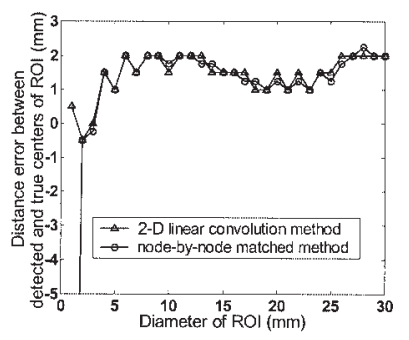

(d)
Fig. 7. Distance error between the detected center of the ROI in the reconstructed image relative to the real location of the circular ROI center is plotted as a function of the diameter of the ROI by use of absorption contrast values of (a) 2.0, (b) 3.0, (c) 4.0, and (d) 5.0 in the target relative to the background. method for calculation of the CNR, we used the background nodes randomly in the image field; thus all the nodes in the background had the same chance to be sampled, but it was not stable when the target was small. Again, this is likely due to problems with a spatially dependent frequency response. Finally, the third method of calculating CNR appeared to be the best, as it included all the nodes in the background image outside of the ROI and corrected the noise weights based on the size ratio of the ROI and the whole image field. This third approach is also easy to implement and provides a reasonable approach for NIR image analysis.

Based on Fig. 5(c) from the third method, we cre-

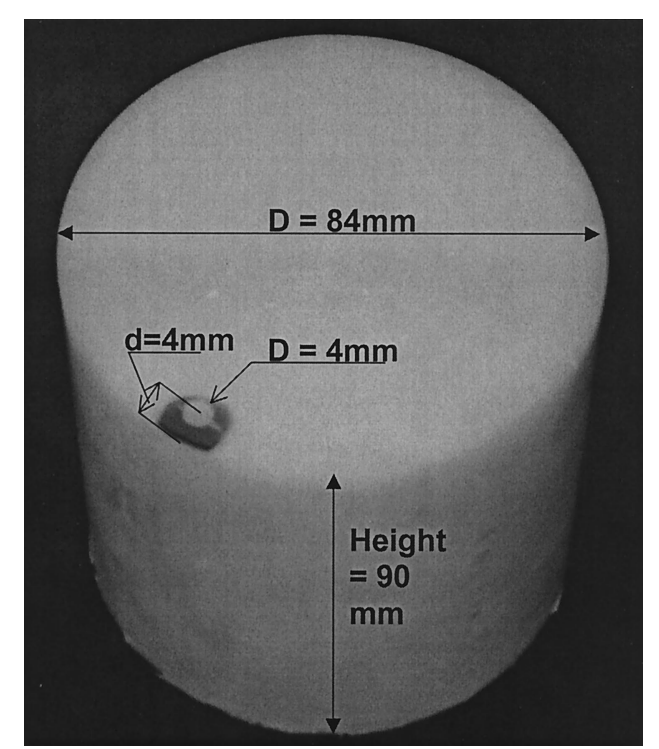

Fig. 8. Photograph of the phantom used in our study. The 4-mm-diameter hole is filled with an Intralipid and ink solution with a variable absorption contrast from $2: 1$ to $9: 1$ and a similar scattering coefficient. Note that the 4-mm hole is outlined with dark ink to make it visible, but this ink on the upper surface of the phantom does not affect the image quality of the interior of the phantom. 


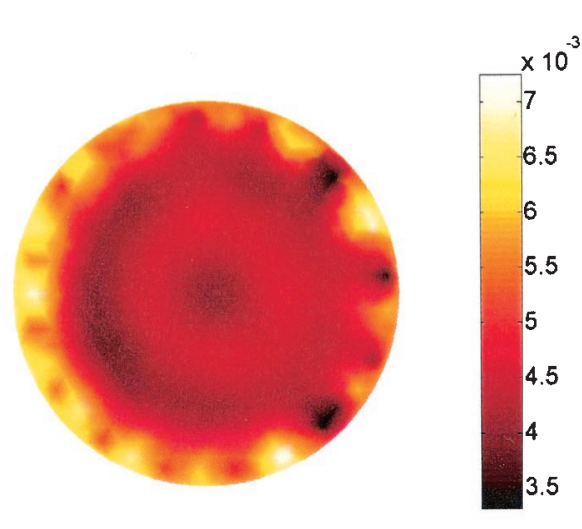

(a)

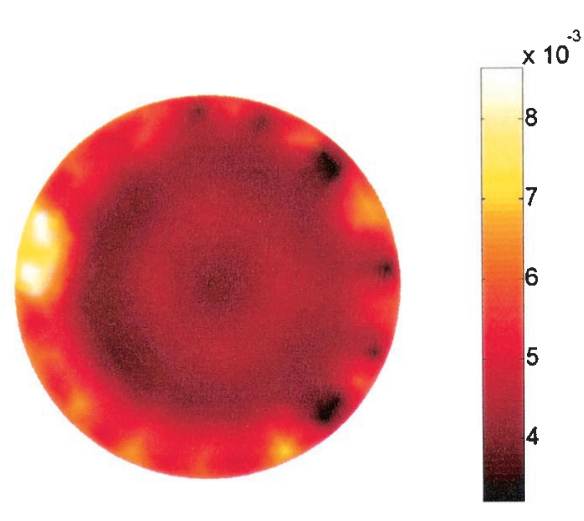

(c)

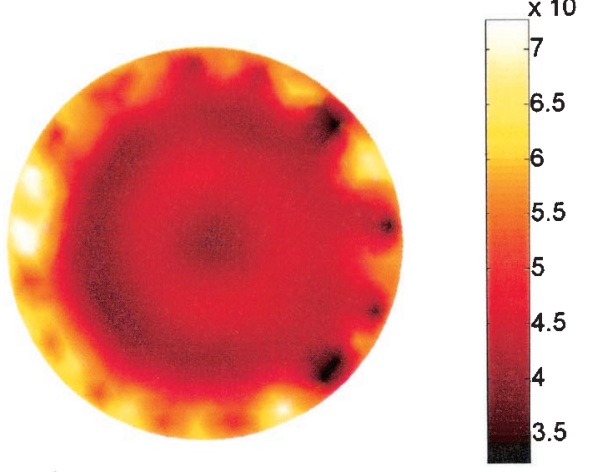

(b)

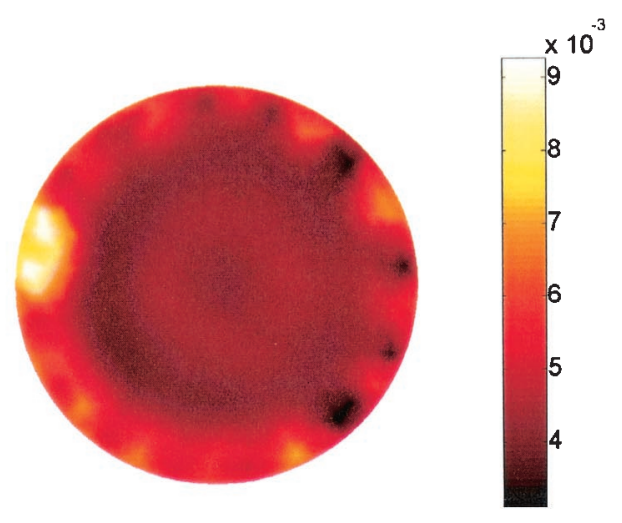

(d)

Fig. 9. Reconstructed images of the absorption coefficient $\left(\mu_{a}\right)$, with the object size of $4 \mathrm{~mm}$ in diameter and the absorption contrast equal to (a) $2: 1$, (b) 4:1, (c) 6:1, and (d) 9:1.

ated Fig. 5(d) using the threshold value of 4 for the lower limit on CNR. The blue regions represent the undetectable conditions (CNR less than 4) and the red regions represent the detectable conditions (CNR greater than 4). From the data in Fig. 5(c) we established that the spatial resolution of the NIR imaging system was $4 \mathrm{~mm}$ in diameter when the contrast is high (i.e., greater than or equal to a contrast of 4.4), and this was established by theoretical simulation and experimental verification. This is interesting in that the system noise level did not appear to have much of an affect on this detection limit, indicating that the lower size limit is perhaps more of a function of the physical phenomenon of how light is diffused out in a highly scattering medium such as tissue. Therefore, if the contrast between the object and the background is high enough, the minimum-detectable size of a target object depends on the physical characteristics of the imaging system and the reconstruction method, but not on the noise of the imaging system or the object characteristics. Also, the contrast resolution (i.e., contrast required for detection at low resolution) was 1.4 when the diameter of the target was large (i.e., greater than or equal to $17 \mathrm{~mm}$ ) with $1 \%$ zero-mean Gaussian noise and $1^{\circ}$ phase shift added. This latter observation would indicate that lower-contrast regions that are large would be difficult to detect when the contrast is less than 1.4 relative to the background; however, this is likely the regime where this CNR threshold of 4 is breaking down. Further analysis of detectability of large objects with a CNR lower than 4 should be performed with multiple observers to determine what the lower limit should really be in this region. We also note that a similar reconstructed ROI image shift of 1-2.5 mm toward the edge of the field occurred in both the simulation and the phantom studies, which is likely an artifact of the strong radially dependent sensitivity that occurs in this type of imaging.

In Fig. 5(d) the contrast-detail curve indicates that the system resolution is limited by both the ROI size and the system noise characteristics. As the contrast increases, the upper left corner of the curve shows the smallest object size that can be detected by the reconstruction system or the spatial resolution of the system. Then, as the ROI size increases, the lower right corner of the curve indicates the smallest 
contrast in object-to-background levels that can be detected by the system. This latter definition is often called the contrast resolution of the system. The spatial resolution and contrast resolution are dependent on the experimential setting of the detection threshold. In our study, the contrast-detail curves are all developed based on the assumption that the detection threshold of the CNR is a value of 4.0. Again, although this number is a useful threshold value and agrees with our experience in small objects, it is likely that it could be considerably lower when one is working on the lower right portion of the contrast-detail curve, where objects are large and the contrast is low. In fact, this CNR value is likely a nonlinear function in itself, which is dependent on object size, contrast, and features of the image reconstruction algorithm. Thus, to complete this study, we chose a CNR value of 4 as a practical working number and have begun a follow-up study to further define how the CNR varied with object and image reconstruction characteristics.

As shown in Fig. 5, the node-by-node matched and 2-D linear convolution methods have similar results for the detection of the ROI location in the reconstructed image. Neither of the two methods was able to detect the ROI location when the diameter of the object being imaged was less than $3 \mathrm{~mm}$. While the diameter of the target increases, the reconstructed positional error range was near 1-2 $\mathrm{mm}$ toward the field edge. Meanwhile, the location errors have no obvious change as the absorption contrast changes from 1.4 (the contrast resolution of our imaging system) to 5 if the diameter is the same. The 2-D linear convolution method appears optimal for the purposes of our system, mainly because it is significantly faster than a random or systematic search algorithm, especially when the size of the target is small.

The lower spatial resolution limit at high contrast levels was determined to be near $4 \mathrm{~mm}$, and we established this by both theoretical simulation and experimental verification using detectability of round objects. This is interesting in that the system noise level did not appear to have much of effect on this detection limit, indicating that the lower size limit is perhaps more of a function of the physics of how light is blurred out in a diffusing medium such as this. Therefore, if the contrast between the object and the background is high enough, the minimum-detectable size of a target object depends on only the physical characteristics of the imaging system and the reconstructed method, such as the width of the laser Guassian beam and the mesh used for reconstruction, but not the noise of the imaging system or measured object. Also, we note that a similar reconstructed ROI image shift of 1-2.5 mm toward the edge of the field occurred in both the simulation and the phantom studies (see Fig. 7), which is likely an artifact of the strong radially dependent sensitivity that occurs in this type of imaging. The amount and direction of the object shifts in the reconstructed images depend on the true position of the target. If the heteroge- neity is placed near the edge of the field, the ROI in the reconstructed images is close to the edge; on the other hand, the ROI shifts toward the center if the target is near the center.

\section{Conclusions}

In our simulation, we calculated the CNR by choosing the background areas including (i) ROIs sampled at the same radial distance in the image, (ii) randomly sampled ROIs of the same size as the original, and (iii) all the available background. According to the comparison, the third method appears to be optimal for NIR tomography because it includes the nodes closest to the field edge. This approach contributed higher background noise and is more stable than the other two methods, especially when the target is small. On the basis of the CNR defined by the third calculation method and a system decision threshold with a CNR of 4 , we find that the NIR imaging system spatial resolution is $4 \mathrm{~mm}$ in diameter and the contrast resolution is 1.4 .

Two approaches were tested to find the location and size of the object based on maximal CNR, including a random node-by-node matching process and a 2-D linear convolution method. Although the results from these two methods are similar, the convolution method is faster than the node-by-node matching process.

Finally, the experimental study on a phantom with similar size and optical properties as in our simulation indicated a series of similar reconstructed images and system spatial resolution. Future plans will involve implementation of this ROI detection tool with our NIR prototype imaging system. The assessment and validation of the threshold CNR value of 4.0 needs to be addressed through multiple observer sessions where images of different size, and contrast levels are examined. Furthermore, because only a single anomaly was used in this study, we would like to upgrade this tool set to diagnose tissue with multiple anomalies. It is also useful to investigate simulated or experimental objects with highly heterogeneous backgrounds such as breast tissue. Future studies should help address these various complex issues associated with NIR as a new and promising imaging modality for physiology and pathology.

This research was sponsored by the National Institutes of Health through grants PO1CA80139 and RO1CA69544.

\section{References}

1. M. A. Franceschini, K. T. Moesta, S. Fantini, G. Gaida, E. Gratton, H. Jess, W. W. Mantulin, M. Seeber, P. M. Schlag, and M. Kaschke, "Frequency-domain techniques enhance optical mammography: initial clinical results," Proc. Natl. Acad. Sci. USA 94, 6468-6473,(1997).

2. B. J. Tromberg, O. Coquoz, J. B. Fishkin, T. Pham, E. R. Anderson, J. Butler, M. Cahn, J. D. Gross, V. Venugopalan, and D. Pham, "Non-invasive measurements of breast tissue optical properties using frequency-domain photon migration," Philos. Trans. R. Soc. London Ser. B 352, 661-668 (1997). 
3. V. Ntziachristos and B. Chance, "Probing physiology and molecular function using optical imaging: applications to breast cancer," Breast Cancer Res. 3, 41-46 (2001).

4. B. W. Pogue, S. P. Poplack, T. O. McBride, W. A. Wells, K. S. Osterman, U. L. Osterberg, and K. D. Paulsen, "Quantitative hemoglobin tomography with diffuse near-infrared spectroscopy: pilot results in the breast," Radiology 218, 261-266 (2001).

5. S. Srinivasan, B. W. Pogue, S. Jiang, H. Dehghani, C. Kogel, S. Soho, J. J. Gibson, T. D. Tosteson, S. P. Poplack, and K. D. Paulsen, "Interpreting hemoglobin and water concentration, oxygen saturation, and scattering measured in vivo by nearinfrared breast tomography," Proc. Natl. Acad. Sci. 100, 12349-12354 (2003).

6. C. H. Schmitz, M. Locker, J. M. Lasker, A. H. Hielscher, and R. L. Barbour, "Instrumentation for fast functional optical tomography," Rev. Sci. Instrum. 73, 429-439 (2002).

7. A. E. Cerussi, A. J. Berger, F. Bevilacqua, N. Shah, D. Jakubowski, J. Butler, R. F. Holcombe, and B. J. Tromberg, "Sources of absorption and scattering contrast for nearinfrared optical mammography," Acad. Radiol. 8, 211-218 (2001).

8. N. Shah, A. Cerussi, C. Eker, J. Espinoza, J. Butler, J. Fishkin, R. Hornung, and B. Tromberg, "Noninvasive functional optical spectroscopy of human breast tissue," Proc. Natl. Acad. Sci. USA 98, 4420-4425 (2001).

9. T. D. Tosteson, B. W. Pogue, E. Demidenko, T. O. McBride, and K. D. Paulsen, "Confidence maps and confidence intervals for near infrared images in breast cancer," IEEE Trans. Med. Imaging 18, 1188-1193 (1999).

10. B. W. Pogue, X. Song, T. D. Tosteson, T. O. McBride, S. Jiang, and K. D. Paulsen, "Statistical analysis of non-linearly reconstructed near-infrared tomographic images. Part I. Theory and simulations," IEEE Med. Imaging 21, 755-763 (2002).

11. X. Song, B. W. Pogue, T. D. Tosteson, T. O. McBride, S. Jiang, K. D. Paulsen, "Statistical analysis of non-linearly reconstructed near-infrared tomographic images. Part II. Experimental interpretation," IEEE Med. Imaging 21, 764-772 (2002).
12. B. W. Pogue, T. O. McBride, J. Prewitt, U. L. Osterberg, K. D. Paulsen, "Spatially variant regularization improves diffuse optical tomography," Appl. Opt. 38, 2950-2961 (1999).

13. B. W. Pogue, C. Willscher, T. O. McBride, U. L. Osterberg, and K. D. Paulsen, "Contrast-detail analysis for detection and characterization with near-infrared diffuse tomography," Med. Phys. 27, 2693-2700 (2000).

14. C. R. Hill, J. C. Bamber, and D. O. Cosgrove, "Performance criteria for quantitative ultrasonography and image parameterisation," Clin. Phys. Physiol. Meas. 11(Suppl A), 57-73 (1990).

15. J. Qi, G. J. Klein, and R. H. Huesman, "Image properties of list-mode likelihood reconstruction for a rectangular positron emission mammograph with DOI measurements," IEEE Trans. Nucl. Sci. 48, 1343-1349 (2001).

16. M. M. Doyley, J. B. Weaver, E. E. Van Houten, F. E. Kennedy, and K. D. Paulsen, "Thresholds for detecting and characterizing focal lesions using steady-state magnetic resonance elastography," Med. Phys. 30, 495-504 (2003).

17. K. J. Robinson, C. J. Kotre, and K. Faulkner, "The use of contrast-detail test objects in the optimization of optical density in mammography,” Br. J. Radiol. 68, 277-282 (1995).

18. H. H. Barrett, C. K. Abbey, and E. Clarkson, "Objective assessment of image quality. III. ROC metrics, ideal observers and likelihood-generating functions," J. Opt. Soc. Am. A 15, 1520-1535 (1998).

19. H. Dehghani, B. W. Pogue, S. P. Poplack, and K. D. Paulsen, "Multiwavelength three-dimensional near-infared tomography of the breast: initial simulation, phantom, and clinical results," Appl. Opt. 42, 135-145 (2003).

20. T. O. McBride, B. W. Pogue, S. Jiang, U. L. Osterberg, and K. D. Paulsen, "Development and calibration of a parallel modulated near-infrared tomography system for hemoglobin imaging in vivo," Rev. Sci. Instrum. 72, 1817-1824 (2001).

21. S. Jiang, B. W. Pogue, T. O. McBride, and K. D. Paulsen, "Quantitative analysis of near-infrared tomography: sensitivity to the tissue-simulating pre-calibration phantom," J. Biomed. Opt. 8, 308-315 (2002). 